\title{
Fronto-Limbic effective connectivity as possible predictor of antidepressant response to SSRI administration.
}

Benedetta Vai ${ }^{12}$, Chiara Bulgarelli ${ }^{1}$, Beata R. Godlewska ${ }^{3}$, Philip J. Cowen ${ }^{3}$, Francesco Benedetti ${ }^{1}$, Catherine J. Harmer ${ }^{3}$

1 IRCCS Ospedale San Raffaele, Department of Clinical Neurosciences, Milan, Italy.

2 Department of Human Studies, Libera Università Maria Ss. Assunta, Roma, Italy.

3 University Department of Psychiatry, Warneford Hospital, Oxford, UK .

\section{Corresponding author:}

Dr. Benedetta Vai

Department of Clinical Neurosciences

Istituto Scientifico Ospedale San Raffaele

San Raffaele Turro

Via Stamira d'Ancona 20

Milano

Italy

Tel $+39 / 02 / 26433156$

Fax +39/02/26433265

E-mail b.vai@hotmail.it 


\begin{abstract}
The timely selection of the optimal treatment for depressed patients is critical to improve remission rates. The detection of pre-treatment variables able to predict differential treatment response may provide novel approaches for treatment selection. Selective serotonin reuptake inhibitors (SSRIs) modulate the frontolimbic functional response and connectivity, an effect preceding the overt clinical antidepressant effects. Here we investigated whether the cortico-limbic connectivity associated with emotional bias measured before SSRI administration predicts the efficacy of antidepressant treatment in MDD patients. $\mathrm{FMRI}$ and Dynamic Causal Modeling (DCM) were combined to study if effective connectivity might differentiate healthy controls $(\mathrm{HC})$ and patients affected by major depression who later responded (RMDD, $n=21)$, or failed to respond ( $\mathrm{RRMDD}, \mathrm{n}=12$ ), to 6 weeks of escitalopram administration. Sixteen DCMs exploring connectivity between anterior cingulate cortex (ACC), ventrolateral prefrontal cortex (VLPFC), Amygdala (Amy), and fusiform gyrus (FG) were constructed. Analyses revealed that nRMDD had reduced endogenous connectivity from Amy to VLPFC and to ACC, with an increased connectivity and modulation of the ACC to Amy connectivity when processing of fearful emotional stimuli compared to HC. RMDD and HC did not significantly differ among themselves. Pre-treatment effective connectivity in fronto-limbic circuitry could be an important factor affecting antidepressant response, and highlight the mechanisms which may be involved in recovery from depression. These results suggest that fronto-limbic connectivity might provide a neural biomarker to predict the clinical outcome to SSRIs administration in major depression.
\end{abstract}

Key words: Dynamic causal modeling, BOLD fMRI, Depression, SSRI, Emotion. 


\section{Introduction}

Mood-congruent negative biases in processing emotional information have been suggested as one of the core features which parallel and contribute to the development and maintenance of depressive states (Beck, 2008) by influencing evaluative processes, social judgment, decision making, attention, and memory (Murphy et al., 1999). Previous studies have demonstrated that depressed patients show perception biases in processing negative emotional facial expressions compared to healthy controls (Bouhuys et al., 1995; Gur et al., 1992). Furthermore, the early reversal of these negative emotional biases could mediate the therapeutic effect of antidepressant drugs (Harmer et al., 2009a; Victor et al., 2010, 2013). Several neuroimaging studies helped to define the neural circuitry engaged in emotional processing. The amygdala (Amy) is a critical node in this network, and is necessary in perceiving stimuli with affective salience, mainly for negative environmental stimuli (Amaral and Price, 1984; LeDoux, 2000), and is activated by emotional memories (Cahill et al., 1996; Hamann et al., 1999). This structure is interconnected with other regions, such as the ventrolateral prefrontal cortex (VLPFC) and anterior cingulate cortex (ACC), which are involved in the attribution of emotional salience to stimuli and in active self-regulation regulation of affective states by modulating Amy reactivity (Beauregard et al., 2001; Ochsner et al., 2004; Phan et al., 2005; Phillips et al., 2003a; Schaefer et al., 2002; Urry et al., 2006). Both these frontal regions are recruited and interact in facial expression processing, these stimuli are initially elaborated by the visual cortex and fusiform gyrus (FG) (Fusar-Poli et al., 2009).

In depression, abnormalities in this functional network have been consistently demonstrated. The most replicated findings reveal increased activation of the Amy and medial prefrontal cortex, and a reduced connectivity between these regions in response to negative emotional stimuli (Davey et al., 2011; Delvecchio et al., 2012; Hamilton et al., 2012; Victor et al., 2012). These findings could reflect a dysfunctional regulation of subcortical limbic activity by cortical areas, which may contribute to the moodcongruent bias in MDD pathophysiology (Rive et al., 2013). This hypothesis is in line with several MRI studies which demonstrated abnormal functional and structural connectivity between frontal and limbic regions in depressed patients compared to controls (Dichter et al., 2014; Shizukuishi et al., 2013).

The regulation of the fronto-limbic circuitry is modulated by serotoninergic transmission: previous studies showed that the activity and functional connectivity in this circuitry was influenced by polymorphisms of the serotonin transporter gene (Dannlowski et al., 2010; Hariri et al., 2002; Klucken et al., 2013; Volman et al., 2013) and by challenges with serotonin reuptake inhibitors (SSRIs) (Grady et al., 2013; Harmer et al., 2006; McCabe et al., 2010; Murphy et al., 2009a; Norbury et al., 2007; Outhred et al., 2013; Phillips et al., 2015; Windischberger et al., 2010). Moreover, depression recovery has been consistently associated with 
functional activity in fronto-limbic structures such as the ACC, medial prefrontal cortex, DLPFC and Amy (Canli et al., 2004; Hamann, 2005; Maddock et al., 2003): before treatment, higher metabolic rates/functional response during passive emotional processing or response inhibition tasks in ACC and Amy were able to predict antidepressant response and remission (Chen et al., 2007; Langenecker et al., 2007; Mayberg et al., 1997; Seminowicz et al., 2004), whereas a lower pre-treatment activity in the ventrolateral prefrontal cortex during the down-regulation of positive emotion was associated with better response to SSRI in depressed patients (Light et al., 2011). Furthermore, a higher connectivity within cortico-limbic networks both, in unipolar and in bipolar depressed patients, was induced by treatment (Anand et al., 2007; Anand et al., 2005; Vai et al., 2015a) and a higher functional resting state connectivity predicted antidepressant response in unipolar depressed patients (Kozel et al., 2011).

The timely selection of the optimal treatment for depressed patients is critical to improving remission rates. The identification of reliable biomarkers, especially the detection of pre-treatment variables that predict differential treatment response, may provide novel approaches for treatment selection (Phillips et al., 2015). However, the prediction offered by cortico-limbic effective connectivity has not been examined despite the hypothesis that depression and response to treatment may best be considered as a network disorder (Vai et al., 2014).

Differences at the baseline neural functional response between depressed patients support the hypothesis that responders or not to antidepressant treatment may differ in terms of neurobiological characteristics (Chen et al., 2007; Gillin et al., 2001; Langenecker et al., 2007; Mayberg et al., 1997; Seminowicz et al., 2004; Wu et al., 1999; Wu et al., 2001)

Therefore, the aim of the present study was to assess whether cortico-limbic connectivity associated with emotional bias predicted the efficacy of antidepressant SSRI treatment in major depressed patients.

Connectivity was explored by combining conventional fMRI with a technique that allows the investigation of causal relationships between regions (Dynamic Causal Modeling, DCM) (Friston et al., 2003). DCM offers a deep comprehension of the connectivity, thanks to the possibility of modeling causality between regions (this aspect mainly differs the effective connectivity from functional one, which does not allow to infer direction). Moreover, DCM estimates how a region causes dynamics in another area via synaptic connections, independently by the experimental condition (intrinsic/endogenous connections), and how these connection change under the influence of external perturbations (i.e. emotional processing of fearful faces, named as modulatory effects) (Stephan et al., 2010).

Specifically, we hypothesized that an increased bottom-up connectivity from Amy and prefrontal cortex could contribute to a better response in terms to depressive recovery. This effect could underlie to a higher 
pre-treatment recruitment of cortical control on subcortical activity in responders, resulting into a more efficient top-down regulation. This was suggested by previous studies which showed a higher connectivity within cortico-limbic networks as pre-treatment neurobiological underpinnings of response during resting state (Kozel et al., 2011) and as neural correlate of antidepressant efficacy (Anand et al., 2007; Anand et al., 2005; Vai et al., 2015a).

\section{Experimental procedures}

\subsection{Participants}

Thirty-three patients with a diagnosis of major depression disorder (MDD) with a current major depressive episode without psychotic features and 31 healthy controls $(\mathrm{HC})$ were studied. All participants were assessed for the presence of current and past psychiatric disorder with the Structured Clinical Interview for DSM-IV (Spitzer, 1995). The depressed patients met criteria for a primary diagnosis of major depressive disorder.

Exclusion criteria for the study were the following. For depressed patients: suffering from psychosis or substance dependence as defined by DSM-IV; being at clinically significant risk of suicidal behavior; having contraindications to escitalopram treatment or being treated with psychotropic medication $<3$ weeks before the study ( 5 weeks in the case of fluoxetine). For healthy volunteers: current or past history of Axis I disorder as defined by DSM-IV. For both groups: major somatic or neurological disorders; pregnancy or breast-feeding; contraindications to magnetic resonance imaging or concurrent medication, which could alter emotional processing. The study was approved by Oxford Research Ethics Committee and all participants gave written informed consent. Participants were reimbursed for their time and any other expenses.

\subsection{Study design and drug treatment}

Patients received $10 \mathrm{mg}$ escitalopram each morning for $5 / 6$ weeks. Depressed patients were scanned before the SSRI administration. Mood was assessed using Hamilton Depression Ratings Scale (Hamilton, 1960), immediately before starting treatment, after one week of treatment and after $5 / 6$ weeks. After the scan all patients were offered treatment openly with escitalopram according to usual clinical practice. Response to the treatment was classified as a reduction of 50\% to the Hamilton Rating Scale for Depression after 5-6 weeks of SSRI administration. The healthy controls were scanned without any pre-treatment. Their mood ratings were carried out just before the scan.

\subsection{Functional MRI data acquisition and experimental task}


Functional MRI data were acquired in a total of 64 participants on a 3T Siemens TIM TRIO (Siemens AG, Erlangen, Germany), fitted with a body transmit coil and 12-channel head receive coil, located at the University of Oxford, Centre for Clinical Magnetic Resonance Research. Functional MRI data were acquired with a voxel resolution of $3 \times 3 \times 3.5 \mathrm{~mm}, T R / T E / F A=2000 \mathrm{~ms} / 28 \mathrm{~ms} / 87 \mathrm{o}$. A total of 255 volumes were acquired in an experiment lasting $8.5 \mathrm{~min}$. Field maps were acquired using a dual echo 2D gradient echo sequence with echos at 5.19 and $7.65 \mathrm{~ms}$ and a repetition time of $444 \mathrm{~ms}$. Data were acquired on a $64 \times 64 \times 40$ grid, with a voxel resolution of $3 \mathrm{~mm}$ isotropic.

During $\mathrm{fMRI}$ scanning, participants completed a simple gender discrimination task involving the rapid presentation of fearful and happy facial expressions. In this task, nine 30-s blocks of a baseline fixation cross (condition A) were interleaved with eight 30-s blocks of the emotional task [four blocks of fear (condition B) and four blocks of happy (condition C)]. During each emotional block, participants viewed 10 emotional faces (five female) all derived from a standard set of pictures of facial affect (Matsumoto, 1988). Each face was presented for $100 \mathrm{~ms}$ and subjects were asked to report the gender of the face via a MRI compatible key pad. To ensure participants remained focused during the task within block inter-stimulus intervals (ISI) ranged between 2500 and $2900 \mathrm{~ms}$ (mean ISI = $2900 \mathrm{~ms}$ ). To reduce potential carry-over effects, cycles of alternation between conditions were counterbalanced across subjects. Thus, during the course of the 8.5-min experiment, half of the subjects completed the following order: ABACABACABACABACA, the remaining subjects ACABACABACABACABA. Stimuli were presented on a personal computer using E-Prime (version 1.0; Psychology Software Tools Inc., USA) and a cloned projection displayed to participants on an opaque screen located at the head of the scanner bore, which subjects viewed using angled mirrors. Subject responses were made via an MRI-compatible keypad. Stimulus presentation/subject button presses were registered and time-locked to fMRI data using E-Prime. Both accuracy (correct gender discrimination) and reaction times were recorded. Immediately before scanning, all subjects received training with another set of stimuli to ensure they fully understood the requirements of the task.

\subsection{Image processing and Second level fMRI Analyses}

All images were analyzed using Statistical Parametric Mapping software (SPM12, http://www.fil.ion.ucl.ac.uk/spm/software/spm12/). Scans were corrected for slice timing and realigned for head movements. Images were then normalized to a standard EPI template volume based on the Montreal Neurological Institute (MNI) reference brain, and smoothed using a 10-mm full-width at half-maximum isotropic Gaussian kernel. The evoked hemodynamic responses were modeled as a delta function convolved with a hemodynamic response and its temporal derivative within the context of the General 
Linear Model (GLM). Thereby isolating regions engaged in the emotional processing of fear, at individual level we subtracted the happy face condition from the fearful one $(p<0.001)$.

We were interested in comparing the pre-treatment DCM parameters in patients affected by major depression who later responded (RMDD), or failed to respond (nRMDD) to 5/6 weeks of SSRI administration and HC. In order to extract time series (Volume of Interest, VOI) in Amy, VLPFC, ACC and FG to perform DCM analyses, a one-sample t-test $(p<0.01)$ were used to identify the peaks of maximum activation for the a priori selected VOI (see Introduction). A two sample t-test was performed in order to explore a possible effect of response on neural response. Group-level analyses were based on the single-subject contrasted images, obtained by subtracting happy face condition to fearful one.

\subsection{Volume of Interest}

We selected a priori volumes of interest for DCM (Amy, VLPFC, ACC, FG) by considering their relevance in facial and emotional processing (Dima et al., 2011; Fairhall and Ishai, 2007; Stein et al., 2007), and antidepressant response (see introduction). Seed regions were identified using Wake Forest PickAtlas software (http://www.fmri.wfubmc.edu).

To account for individual differences, we extracted principal eigenvariates in $8 \mathrm{~mm}$ spheres centered in regions mentioned above. The exact location of the $8 \mathrm{~mm}$ sphere was based upon the local maxima of the subject-specific statistical maps within $8 \mathrm{~mm}$ of the group-maxima univariate analysis (one sample t-test in section 2.4). MNI coordinates were 4632 -2 for VLPFC, 22020 for ACC, and 46 -38 -22 for FG, while Amy was considered as a whole. Our DCM analyses was restricted to the right hemisphere, because our previous study conducted in depressed patients showed that escitalopram lowers fear responses in the right Amy rather than bilaterally (Godlewska et al., 2012a) and other studies in healthy volunteers confirmed that antidepressant treatment mainly induce changes in the right hemisphere during emotional processing (Harmer et al., 2006; Murphy et al., 2009b; Rawlings et al., 2010). Moreover, the general predominance of this hemisphere in processing of emotional faces has been demonstrated in different studies (Dima et al., 2011; Fairhall and Ishai, 2007). The higher involvement of right hemisphere was also confirmed by the results of our second level analyses (see Results Section).

\subsection{Dynamic Causal Modeling: Structural and parametric analyses}

DCM assess the dynamic behavior of specific brain regions regarding their causal relationships under the influence of external perturbations, such as the applied experimental conditions, allowing to describe (i) how the present state of one neuronal population causes dynamics (i.e., rate of change) in another via synaptic connections (intrinsic connections) and (ii) how these interactions change under the influence of 
external perturbations (i.e., how experimental manipulations as the negative emotional valence of faces modulate the strength of endogenous connections; modulatory effects) (Stephan et al., 2010) and (iii) driving inputs, that could be considered as direct influences of the stimuli on the neural activity of involved regions. After specifying and estimating different models, Bayesian Model Averaging (BMA) was performed to obtained DCMs' parameters in order to compare the strength of theme among groups. BMA summarizes quantitatively parameter estimates of intrinsic connections, modulatory effects and driving inputs and was performed using the random effects (Stephan et al., 2010). Furthermore, the context-sensitive connectivity between regions could obtained by summing endogenous and modulatory parameters. BMA averages each parameter across subjects and across models, so that the contribution of each model (of each subject) for that parameter is weighted by each model's posterior probability for that subject (Penny et al., 2010; Stephan et al., 2010). This probability is obtained by Bayesian Model Selection. BMS provides the exceedance probability (EP), which is the probability that a model is more likely than any other model in model space and the Protected Exceedance Probability (Protected EP), which quantify the probability that any model is more frequent than others, above and beyond the chance (Rigoux et al., 2014). BMS also computes Bayesian Omnibus Risk (BOR) as a measure of the statistical risk incurred when performing BMS (Rigoux et al., 2014). These results could provide an general evaluation of the strength of the evidence; however, their interpretation could be difficult when considering some limitations of BMS: identifying the best model independently for the different groups does not directly test the hypothesis that the same model describes the different groups, by allowing to check anecdotally whether the results are consistent or not among groups (Rigoux et al., 2014). Furthermore, theoretically, even when underlying models are all identical, natural variations in within-subjects model evidences could induce some model selection errors and it is also possible that a wide within group variability, in terms of models, increases the chance probability of observing (seemingly) different frequency profiles (Rigoux et al., 2014). Thus, in order to explore differences among groups, BMS had been combined with BMA (Sladky et al., 2015a).

We modeled the differential state equations on different seed regions of interest (Amy, VLPFC, ACC, FG in the right hemisphere). Sixteen alternative models with different modulatory effects of fearful emotional faces were constructed with DCM12 (SPM12, Released December 2012. See Fig. 1 and Supplementary materials for model space). All models were defined as bilinear and deterministic. Affective information (faces) could enter the network by directly activating FG and Amy, fixed across all models (Diwadkar et al., 2012; Vai et al., 2015b). In all the proposed models, we fixed forward intrinsic connection from FG to Amy. The connectivity between ACC, VLPFC and Amy was set bidirectional across all the models. This model space based on previous primate and in vivo imaging studies, and represents the bottom-up and top-down flow information in cortico-limbic circuitry (Dima et al., 2011; Diwadkar et al., 2012; Phelps and LeDoux, 2005; Vai et al., 2015b). The 16 models varied for the presence or absence of modulatory effects of emotional processing of fearful faces on the connections between VLPFC, ACC and Amy. 
BMS compared the 16 models separately of each group. After BMA, we included the individual DCM parameters in statistical analyses. Repeated-measures analyses of variance on HAMD scores were performed in the context of the General Linear Model (McCulloch et al., 2008). The main factors of interest were response to treatment and time. DCM parameters were compared between HC, nRMDD and RMDD at the pre-treatment; age and sex were entered as covariates of no interest. We applied Bonferroni-Holm correction to account for multiple comparisons (Holm, 1979). Analyses were performed using a commercially available software (StatSoft Statistica 8.0, Tulsa, OK, USA).

\section{Results}

\subsection{Clinical response}

Clinical and demographic characteristics of the sample are resumed in Table 1. Patients and controls did not significantly differ for age and gender.

Twenty-one (63.6\%) patients responded to treatment (HAM-D scores 50\% reduction), and did not significantly differ from nRMDD $(n=12)$ on any clinical and demographic measure. Patterns of mood change during treatment markedly differed among RMDD and nRMDD (Time: $F=3.37$; d.f. 2,56; $p=0.042$; Response: $F=12.97$; d.f. 1,28; $p<0.001$; Interaction: $F=19.22$; d.f. 2,56; $p<0.0001 ;$ Fig. 2): nRMDD however achieved a significant improvement after 5/6 weeks (post hoc Fisher's least significant difference: final scores vs pretreatment $p=0.002$ ), while responders showed a progressive amelioration during the treatment (post hoc: intermediate scores vs pre-treatment $p<0.001$; final scores both vs pre-treatment in RMDD and vs non responders final scores $p<0.0001)$. RMDD and $\mathrm{nRMDD}$ were not significantly different at the pre-treatment. Notably, 95\% Confidence Intervals at 5/6 weeks of treatment suggest large differences between RMDD and nRMDD at final HAMD scores, with no overlap between the two groups.

\section{2 fMRI Activity Data and Dynamic Causal Modeling}

One sample t-test performed on $\mathrm{FMRI}$ data in $\mathrm{HC}$, RMDD and nRMDD showed that the task significantly activated our seed regions (Fig. 1): VLPFC (peak MNI coordinates $4632-2 ; \mathrm{T}=3.77 ; \mathrm{Z}=3.57 ; \mathrm{p}<0.001$ ), ACC (peak MNI coordinates 220 20; T= 2.46; z=2.40; $p<0.01$ ), Amy (peak MNI coordinates $28-4-16$; $T=3.65$; $Z=3.55 ; p<0.001$ ) and FG (peak MNI coordinates $46-38-22 ; T=4.24 ; Z=3.96 ; p<0.001$ ). Results of one sample t-tests are reported in Table 1 in Supplementary Materials. No significant differences between RMDD and nRMDD survived to correction for multiple comparison.

Random effect BMSs showed that the $8^{\text {th }}$ model was the winning one in $\mathrm{HC}$ ( $\mathrm{BOR}=0.99$; Protected $\mathrm{EP}=0.065$; $E P=0.24)$, the $9^{\text {th }}$ in $R M D D(B O R=0.99$; Protected $E P=0.14 ; E P=0.41)$ and the $13^{\text {th }}$ in $n R M D D(B O R=0.84$; 
Protected $\mathrm{EP}=0.067 ; \mathrm{EP}=0.3)$. As described in the Methods section, all the models showed forward intrinsic connection from FG to Amy, while connectivity between ACC, VLPFC and Amy was set bidirectional. Both the $8^{\text {th }}$ and the $9^{\text {th }}$ models present modulatory effects on the bottom up connection from Amy to VLPFC; additionally, the $8^{\text {th }}$ has a modulatory effect on connection from ACC to Amy, whereas the $9^{\text {th }}$ on the bottom up connection from Amy to ACC. The $13^{\text {th }}$, which is the winning model for nRMDD, has bidirectional modulatory effects on the connection Amy-ACC and a modulatory effect on the connection from Amy to VLPFC. BMS indexes revealed minor structural differences among models in each groups.

After extracting DCM parameters, by averaging all models (BMA), analyses performed at the pre-treatment revealed significant differences between $\mathrm{HC}$, RMDD and nRMDD (Table 2, Fig. 3) for the endogenous connections from Amy to ACC ( $F=5.91$, uncorrected $p=0.005$,), from Amy to VLPFC ( $F=4.84$, uncorrected $p=0.011)$, in the modulatory effect from $A C C$ to Amy $(F=6.82, p=0.002$, surviving Bonferroni-Holm correction for multiple comparisons), and in the connectivity during emotional processing by combining endogenous and modulatory parameters $(F=6.53, p=0.003$, Bonferroni-Holm corrected). Post-hoc tests revealed that $\mathrm{nRMDD}$ had a reduced endogenous connection from Amy to prefrontal cortexes compared to HC $(p<0.05)$. These connections are inhibitory in $n R M D D$, whereas $H C$ and RMDD showed excitatory bottom-up connections. nRMDD had an increased and excitatory modulatory effect from ACC to Amy compared to $\mathrm{HC}(\mathrm{p}<0.05)$, who instead presented inhibitory top-down control from ACC to AmY. RMDD and HC did not significantly differ among themselves. Furthermore, the connectivity during emotional processing, endogenous summed to modulatory parameters, was significantly increased in nRMDD compared to $\mathrm{HC}$, who instead showed a negative effective connectivity $(p<0.05)$. An additional confirmatory analysis about a possible relationship between FC parameters, detected as different among groups, and clinical improvement was performed; methods and results of the analysis could be found in the Supplementary Materials.

\section{Discussion}


Our study revealed that the effective connectivity within fronto-limbic circuitry is associated with later clinical response to 6 weeks of escitalopram treatment. nRMDD showed and increased connectivity from ACC to Amy during negative emotional processing compared to HC and RMDD at pre-treatment. This effect seems to be driven from a higher modulatory effect of the task in nRMDD compared to HC. Furthermore, nRMDD was characterized by a reduced bottom-up connectivity from Amy to prefrontal areas compared to HC (perigenual ACC and VLPFC). RMDD showed a pattern of cortico-limbic connectivity parameters comparable to that of $\mathrm{HC}$. This pattern of results supports the hypothesis that non-responders to antidepressant treatments may diverge from responders in terms of neurobiological characteristics prior to treatment. Previous studies pointed out that the baseline functional characteristics of Amy, ACC, and VLPFC predict antidepressant response (Chen et al., 2007; Langenecker et al., 2007; Mayberg et al., 1997). The current pattern of results suggests that the connectivity between these key nodes may also be critical for treatment success.

Amy, ACC, and VLPFC play crucial roles in the experience of emotions and in the cognitive generation of affect, and in regulating autonomic responses to stressful events, assigning emotional valence to internal and external stimuli, and emotional expression (Davidson et al., 2002; Pizzagalli, 2011). Depression is characterized by a reduced functional and effective connectivity between medial prefrontal cortex and Amy (Davey et al., 2011; Delvecchio et al., 2012; Hamilton et al., 2012; Victor et al., 2012), and over-activation of the Amy in response to negative stimuli. These abnormalities have been proposed to result in a restriction of the range of emotions identifiable and experienced and in an increased tendency to identify stimuli as emotional connected, but with a bias toward negative rather than positive emotions. This may then result in the production of depressed mood and anhedonia (Phillips et al., 2003b). SSRIs administration is associated with a normalization of both cortico-limbic activity and connectivity as a reversal of the moodcongruent negative biases detected in depression (Harmer et al., 2009a; Harmer et al., 2009b; Phillips et al., 2015; Victor et al., 2013). SSRIs administration increases the level of 5-HT in the synapses through blocking the reuptake of serotonin. This leads to cascade changes which include the desensitisation of 5-HT autoreceptors, the restoration of function in second messenger systems, and transcription of neurotrophic factors (Stahl, 1998).

Amy, ACC, and VLPFC receive projections from serotonergic neurons, and previous findings confirm that SSRIs act on this circuit, by reducing Amy functional responses to emotional stimuli in MDD patients (Godlewska et al., 2012b), by modulating Amy effective connectivity in healthy subjects (Sladky et al., 2015b), and by inducing a higher connectivity within cortico-limbic networks (Anand et al., 2007; Anand et al., 2005). In terms of restored cortical inhibitory modulation of limbic hyperactivity, an increased top-down connectivity from prefrontal cortex to Amy is a marker of antidepressant response in bipolar disorder (Vai et al., 2015a). Nevertheless, our present study suggests that top-down influences might be biased by the 
pre-treatment status of network connectivity, which acts as a possible predictor of effective response to SSRIs treatment. In particular, neither RMDD nor HC did differ for the connectivity and effect of fearful faces on the top-down connection from ACC to Amy: the connectivity is inhibitory in HC, suggesting an effective control of prefrontal cortex in modulating Amy response to negative stimuli. Nevertheless, nRMDD showed a completely opposite pattern: the connectivity and modulatory effect is not only significantly increased compared to $\mathrm{HC}$, but also qualitatively excitatory. This could interfere with the restoring of the prefrontal control resulting from the SSRI administration in $\mathrm{nRMDD}$; however, in the RMDD group, who did not significantly differ from HC, the SSRI administration could more efficiently improve the prefrontal inhibitory control. This pattern is likely to be observed also in the bottom-up connection from AMY to ACC and VLPFC: in fact, both in HC and in RMDD the endogenous connections are excitatory and significantly different from nRMDD, where this connection has shown to be inhibitory. This could suggest that both in RMDD and in HC groups the Amy positively engages the prefrontal control, whereas an increase activation of Amy is paralleled by a reduced recruitment of prefrontal neural response in the nRMDD group.

These effects might also involve white matter (WM) tracts. Diffusion tensor imaging measures of WM microstructure suggest lower directionality between ventral prefrontal cortex and Amy in depression (Phillips et al., 2015), and directly associated, in terms of pre-treatment status, with a poor antidepressant response (Delorenzo et al., 2013). Genetic factors affecting 5-HT neurotransmission influence WM microstructure in mood disorders (Benedetti et al., 2015), as well as SSRIs (Benedetti et al., 2012; Yoo et al., 2007), with oligodendrocytes actively expressing $5-\mathrm{HT}_{2 \mathrm{~A}}$ receptors throughout their life cycle (Belachew et al., 1998). Further research will clarify if a disrupted structural connectivity parallels the functional dysregulation of the brain networks involved in mood and emotions, thus limit the efficacy of antidepressant treatments in rebalancing cortical and limbic neural responses.

Limitations of the present study, which is correlational in nature, also include issues such as generalizability, possible population stratification, medications over the lifetime and their effects on the observed differences, no placebo control, no evaluation for compliance, varying treatment periods and technical issues related to the specific methods of neuroimaging data analysis. These limitations do not bias the main finding that the clinical antidepressant response to SSRIs administration is directly associated with the pretreatment effective connectivity between the Amy and prefrontal cortex during the processing of implicit negative affective states. In line with these previous findings in depression, the pre-treatment status of the brain could be a major factor affecting antidepressant response, mediated by emotional biases, and mechanisms underlying the functional activity of the brain can be involved in the biological changes needed to recover from depression (Vai et al., 2015a). A reduced and inhibitory endogenous connectivity, as well as an excitatory and increased connectivity and modulation of top-down connections between Amy and 
ventral prefrontal cortex may identify a neurobiological correlate of non-response of SSRIs administration in depressed patients, independently from baseline symptoms severity.

\section{Acknowledgment}

None 


\section{References}

Amaral, D.G., Price, J.L., 1984. Amygdalo-cortical projections in the monkey (Macaca fascicularis). J Comp Neurol 230, 465-496.

Anand, A., Li, Y., Wang, Y., Gardner, K., Lowe, M.J., 2007. Reciprocal effects of antidepressant treatment on activity and connectivity of the mood regulating circuit: an FMRI study. J Neuropsychiatry Clin Neurosci 19, 274-282.

Anand, A., Li, Y., Wang, Y., Wu, J., Gao, S., Bukhari, L., Mathews, V.P., Kalnin, A., Lowe, M.J., 2005. Antidepressant effect on connectivity of the mood-regulating circuit: an FMRI study.

Neuropsychopharmacology 30, 1334-1344.

Beauregard, M., Levesque, J., Bourgouin, P., 2001. Neural correlates of conscious self-regulation of emotion. J Neurosci 21, RC165.

Beck, A.T., 2008. The evolution of the cognitive model of depression and its neurobiological correlates. Am J Psychiatry 165, 969-977.

Belachew, S., Malgrange, B., Rigo, J.M., Rogister, B., Coucke, P., Mazy-Servais, C., Moonen, G., 1998. Developmental regulation of neuroligand-induced responses in cultured oligodendroglia. Neuroreport 9 , 973-980.

Benedetti, F., Bollettini, I., Poletti, S., Locatelli, C., Lorenzi, C., Pirovano, A., Smeraldi, E., Colombo, C., 2015. White matter microstructure in bipolar disorder is influenced by the serotonin transporter gene polymorphism 5-HTTLPR. Genes Brain Behav. 14, 238-250.

Benedetti, F., Giacosa, C., Radaelli, D., Poletti, S., Dallaspezia, S., Pozzi, E., Falini, A., Smeraldi, E., 2012. Widespread changes of white matter microstructure in obsessive-compulsive disorder: Effect of drug status. . Eur. Neuropsychopharmacol.

Bouhuys, A.L., Bloem, G.M., Groothuis, T.G., 1995. Induction of depressed and elated mood by music influences the perception of facial emotional expressions in healthy subjects. J Affect Disord 33, 215-226. Cahill, L., Haier, R.J., Fallon, J., Alkire, M.T., Tang, C., Keator, D., Wu, J., McGaugh, J.L., 1996. Amygdala activity at encoding correlated with long-term, free recall of emotional information. Proc Natl Acad Sci U S A 93, 8016-8021.

Canli, T., Sivers, H., Thomason, M.E., Whitfield-Gabrieli, S., Gabrieli, J.D., Gotlib, I.H., 2004. Brain activation to emotional words in depressed vs healthy subjects. Neuroreport 15, 2585-2588.

Chen, C.H., Ridler, K., Suckling, J., Williams, S., Fu, C.H., Merlo-Pich, E., Bullmore, E., 2007. Brain imaging correlates of depressive symptom severity and predictors of symptom improvement after antidepressant treatment. Biol Psychiatry 62, 407-414.

Dannlowski, U., Konrad, C., Kugel, H., Zwitserlood, P., Domschke, K., Schoning, S., Ohrmann, P., Bauer, J., Pyka, M., Hohoff, C., Zhang, W., Baune, B.T., Heindel, W., Arolt, V., Suslow, T., 2010. Emotion specific modulation of automatic amygdala responses by 5-HTTLPR genotype. Neuroimage 53, 893-898.

Davey, C.G., Allen, N.B., Harrison, B.J., Yucel, M., 2011. Increased amygdala response to positive social feedback in young people with major depressive disorder. Biol Psychiatry 69, 734-741.

Davidson, R.J., Pizzagalli, D., Nitschke, J.B., Putnam, K., 2002. Depression: perspectives from affective neuroscience. Annu. Rev. Psychol. 53, 545-574.

Delorenzo, C., Delaparte, L., Thapa-Chhetry, B., Miller, J.M., Mann, J.J., Parsey, R.V., 2013. Prediction of selective serotonin reuptake inhibitor response using diffusion-weighted MRI. Front Psychiatry 4, 5. Delvecchio, G., Fossati, P., Boyer, P., Brambilla, P., Falkai, P., Gruber, O., Hietala, J., Lawrie, S.M., Martinot, J.L., McIntosh, A.M., Meisenzahl, E., Frangou, S., 2012. Common and distinct neural correlates of emotional processing in Bipolar Disorder and Major Depressive Disorder: a voxel-based meta-analysis of functional magnetic resonance imaging studies. Eur Neuropsychopharmacol 22, 100-113.

Dichter, G.S., Gibbs, D., Smoski, M.J., 2014. A systematic review of relations between resting-state functional-MRI and treatment response in major depressive disorder. J Affect Disord 172C, 8-17. Dima, D., Stephan, K.E., Roiser, J.P., Friston, K.J., Frangou, S., 2011. Effective connectivity during processing of facial affect: evidence for multiple parallel pathways. J Neurosci 31, 14378-14385.

Diwadkar, V.A., Wadehra, S., Pruitt, P., Keshavan, M.S., Rajan, U., Zajac-Benitez, C., Eickhoff, S.B., 2012. Disordered corticolimbic interactions during affective processing in children and adolescents at risk for 
schizophrenia revealed by functional magnetic resonance imaging and dynamic causal modeling. Arch Gen Psychiatry 69, 231-242.

Fairhall, S.L., Ishai, A., 2007. Effective connectivity within the distributed cortical network for face perception. Cereb Cortex 17, 2400-2406.

Friston, K.J., Harrison, L., Penny, W., 2003. Dynamic causal modelling. Neuroimage 19, 1273-1302.

Fusar-Poli, P., Placentino, A., Carletti, F., Landi, P., Allen, P., Surguladze, S., Benedetti, F., Abbamonte, M., Gasparotti, R., Barale, F., Perez, J., McGuire, P., Politi, P., 2009. Functional atlas of emotional faces

processing: a voxel-based meta-analysis of 105 functional magnetic resonance imaging studies. J Psychiatry Neurosci 34, 418-432.

Gillin, J.C., Buchsbaum, M., Wu, J., Clark, C., Bunney, W., Jr., 2001. Sleep deprivation as a model experimental antidepressant treatment: findings from functional brain imaging. Depress Anxiety 14, 37-49. Godlewska, B.R., Norbury, R., Selvaraj, S., Cowen, P.J., Harmer, C.J., 2012a. Short-term SSRI treatment normalises amygdala hyperactivity in depressed patients. Psychol Med 42, 2609-2617.

Godlewska, B.R., Norbury, R., Selvaraj, S., Cowen, P.J., Harmer, C.J., 2012b. Short-term SSRI treatment normalises amygdala hyperactivity in depressed patients. Psychol. Med. 42, 2609-2617.

Grady, C.L., Siebner, H.R., Hornboll, B., Macoveanu, J., Paulson, O.B., Knudsen, G.M., 2013. Acute pharmacologically induced shifts in serotonin availability abolish emotion-selective responses to negative face emotions in distinct brain networks. Eur Neuropsychopharmacol 23, 368-378.

Gur, R.C., Erwin, R.J., Gur, R.E., Zwil, A.S., Heimberg, C., Kraemer, H.C., 1992. Facial emotion discrimination: II. Behavioral findings in depression. Psychiatry Res 42, 241-251.

Hamann, S., 2005. Blue genes: wiring the brain for depression. Nat Neurosci 8, 701-703.

Hamann, S.B., Ely, T.D., Grafton, S.T., Kilts, C.D., 1999. Amygdala activity related to enhanced memory for pleasant and aversive stimuli. Nat Neurosci 2, 289-293.

Hamilton, J.P., Etkin, A., Furman, D.J., Lemus, M.G., Johnson, R.F., Gotlib, I.H., 2012. Functional

neuroimaging of major depressive disorder: a meta-analysis and new integration of base line activation and neural response data. Am J Psychiatry 169, 693-703.

Hamilton, M., 1960. A rating scale for depression. J Neurol Neurosurg Psychiatry 23, 56-62.

Hariri, A.R., Mattay, V.S., Tessitore, A., Kolachana, B., Fera, F., Goldman, D., Egan, M.F., Weinberger, D.R., 2002. Serotonin transporter genetic variation and the response of the human amygdala. Science $297,400-$ 403.

Harmer, C.J., Goodwin, G.M., Cowen, P.J., 2009a. Why do antidepressants take so long to work? A cognitive neuropsychological model of antidepressant drug action. Br J Psychiatry 195, 102-108.

Harmer, C.J., Mackay, C.E., Reid, C.B., Cowen, P.J., Goodwin, G.M., 2006. Antidepressant drug treatment modifies the neural processing of nonconscious threat cues. Biol Psychiatry 59, 816-820.

Harmer, C.J., O'Sullivan, U., Favaron, E., Massey-Chase, R., Ayres, R., Reinecke, A., Goodwin, G.M., Cowen, P.J., 2009b. Effect of acute antidepressant administration on negative affective bias in depressed patients. Am J Psychiatry 166, 1178-1184.

Holm, S., 1979. Holm's sequential bonferroni procedure. Scand J Stat 6, 65-70.

Klucken, T., Wehrum, S., Schweckendiek, J., Merz, C.J., Hennig, J., Vaitl, D., Stark, R., 2013. The 5-HTTLPR polymorphism is associated with altered hemodynamic responses during appetitive conditioning. Hum Brain Mapp 34, 2549-2560.

Kozel, F.A., Rao, U., Lu, H., Nakonezny, P.A., Grannemann, B., McGregor, T., Croarkin, P.E., Mapes, K.S., Tamminga, C.A., Trivedi, M.H., 2011. Functional connectivity of brain structures correlates with treatment outcome in major depressive disorder. Front Psychiatry 2, 7.

Langenecker, S.A., Kennedy, S.E., Guidotti, L.M., Briceno, E.M., Own, L.S., Hooven, T., Young, E.A., Akil, H., Noll, D.C., Zubieta, J.K., 2007. Frontal and limbic activation during inhibitory control predicts treatment response in major depressive disorder. Biol Psychiatry 62, 1272-1280.

LeDoux, J.E., 2000. Emotion circuits in the brain. Annu Rev Neurosci 23, 155-184.

Light, S.N., Heller, A.S., Johnstone, T., Kolden, G.G., Peterson, M.J., Kalin, N.H., Davidson, R.J., 2011.

Reduced right ventrolateral prefrontal cortex activity while inhibiting positive affect is associated with improvement in hedonic capacity after 8 weeks of antidepressant treatment in major depressive disorder. Biol Psychiatry 70, 962-968. 
Maddock, R.J., Garrett, A.S., Buonocore, M.H., 2003. Posterior cingulate cortex activation by emotional words: fMRI evidence from a valence decision task. Hum Brain Mapp 18, 30-41.

Matsumoto, J., Ekman, P. , 1988. Japanese and Caucasian Facial Expressions of Emotion (JACFEE) and Neutral Faces (JACNeuf). San Francisco.

Mayberg, H.S., Brannan, S.K., Mahurin, R.K., Jerabek, P.A., Brickman, J.S., Tekell, J.L., Silva, J.A., McGinnis, S., Glass, T.G., Martin, C.C., Fox, P.T., 1997. Cingulate function in depression: a potential predictor of treatment response. Neuroreport 8, 1057-1061.

McCabe, C., Mishor, Z., Cowen, P.J., Harmer, C.J., 2010. Diminished neural processing of aversive and rewarding stimuli during selective serotonin reuptake inhibitor treatment. Biol Psychiatry 67, 439-445. McCulloch, C.E., Searle, S.R., Neuhaus, J.M., 2008. Generalized, Linear, and Mixed Models. Second Edition. . John Wiley \& Sons, New York.

Murphy, F.C., Sahakian, B.J., Rubinsztein, J.S., Michael, A., Rogers, R.D., Robbins, T.W., Paykel, E.S., 1999. Emotional bias and inhibitory control processes in mania and depression. Psychol Med 29, 1307-1321. Murphy, S.E., Norbury, R., O'Sullivan, U., Cowen, P.J., Harmer, C.J., 2009a. Effect of a single dose of citalopram on amygdala response to emotional faces. Br J Psychiatry 194, 535-540.

Murphy, S.E., Yiend, J., Lester, K.J., Cowen, P.J., Harmer, C.J., 2009b. Short-term serotonergic but not noradrenergic antidepressant administration reduces attentional vigilance to threat in healthy volunteers. Int J Neuropsychopharmacol 12, 169-179.

Norbury, R., Mackay, C.E., Cowen, P.J., Goodwin, G.M., Harmer, C.J., 2007. Short-term antidepressant treatment and facial processing. Functional magnetic resonance imaging study. Br J Psychiatry 190, 531532.

Ochsner, K.N., Ray, R.D., Cooper, J.C., Robertson, E.R., Chopra, S., Gabrieli, J.D., Gross, J.J., 2004. For better or for worse: neural systems supporting the cognitive down- and up-regulation of negative emotion. Neuroimage 23, 483-499.

Outhred, T., Hawkshead, B.E., Wager, T.D., Das, P., Malhi, G.S., Kemp, A.H., 2013. Acute neural effects of selective serotonin reuptake inhibitors versus noradrenaline reuptake inhibitors on emotion processing: Implications for differential treatment efficacy. Neurosci Biobehav Rev 37, 1786-1800.

Phan, K.L., Fitzgerald, D.A., Nathan, P.J., Moore, G.J., Uhde, T.W., Tancer, M.E., 2005. Neural substrates for voluntary suppression of negative affect: a functional magnetic resonance imaging study. Biol Psychiatry 57, 210-219.

Phelps, E.A., LeDoux, J.E., 2005. Contributions of the amygdala to emotion processing: from animal models to human behavior. Neuron 48, 175-187.

Phillips, M.L., Chase, H.W., Sheline, Y.I., Etkin, A., Almeida, J.R., Deckersbach, T., Trivedi, M.H., 2015. Identifying predictors, moderators, and mediators of antidepressant response in major depressive disorder: neuroimaging approaches. Am J Psychiatry 172, 124-138.

Phillips, M.L., Drevets, W.C., Rauch, S.L., Lane, R., 2003a. Neurobiology of emotion perception I: The neural basis of normal emotion perception. Biol Psychiatry 54, 504-514.

Phillips, M.L., Drevets, W.C., Rauch, S.L., Lane, R., 2003b. Neurobiology of emotion perception II: Implications for major psychiatric disorders. Biol Psychiatry 54, 515-528.

Pizzagalli, D.A., 2011. Frontocingulate dysfunction in depression: toward biomarkers of treatment response. Neuropsychopharmacology 36, 183-206.

Rawlings, N.B., Norbury, R., Cowen, P.J., Harmer, C.J., 2010. A single dose of mirtazapine modulates neural responses to emotional faces in healthy people. Psychopharmacology (Berl) 212, 625-634.

Rigoux, L., Stephan, K.E., Friston, K.J., Daunizeau, J., 2014. Bayesian model selection for group studies revisited. Neuroimage 84, 971-985.

Rive, M.M., van Rooijen, G., Veltman, D.J., Phillips, M.L., Schene, A.H., Ruhe, H.G., 2013. Neural correlates of dysfunctional emotion regulation in major depressive disorder. A systematic review of neuroimaging studies. Neurosci Biobehav Rev 37, 2529-2553.

Schaefer, S.M., Jackson, D.C., Davidson, R.J., Aguirre, G.K., Kimberg, D.Y., Thompson-Schill, S.L., 2002. Modulation of amygdalar activity by the conscious regulation of negative emotion. J Cogn Neurosci 14, 913921.

Seminowicz, D.A., Mayberg, H.S., McIntosh, A.R., Goldapple, K., Kennedy, S., Segal, Z., Rafi-Tari, S., 2004. Limbic-frontal circuitry in major depression: a path modeling metanalysis. Neuroimage 22, 409-418. 
Shizukuishi, T., Abe, O., Aoki, S., 2013. Diffusion tensor imaging analysis for psychiatric disorders. Magn Reson Med Sci 12, 153-159.

Sladky, R., Hoflich, A., Kublbock, M., Kraus, C., Baldinger, P., Moser, E., Lanzenberger, R., Windischberger, C., 2015a. Disrupted effective connectivity between the amygdala and orbitofrontal cortex in social anxiety disorder during emotion discrimination revealed by dynamic causal modeling for FMRI. Cereb Cortex 25, 895-903.

Sladky, R., Spies, M., Hoffmann, A., Kranz, G., Hummer, A., Gryglewski, G., Lanzenberger, R., Windischberger, C., Kasper, S., 2015b. (S)-citalopram influences amygdala modulation in healthy subjects: a randomized placebo-controlled double-blind fMRI study using dynamic causal modeling. Neuroimage 108, 243-250.

Spitzer, R.L., Williams, J.B.W., Gibbon, M., First, M.B. , 1995. Structured Clinical Interview for DSM-1V (SCID), New York.

Stahl, S.M., 1998. Mechanism of action of serotonin selective reuptake inhibitors. Serotonin receptors and pathways mediate therapeutic effects and side effects. J Affect Disord 51, 215-235.

Stein, J.L., Wiedholz, L.M., Bassett, D.S., Weinberger, D.R., Zink, C.F., Mattay, V.S., Meyer-Lindenberg, A., 2007. A validated network of effective amygdala connectivity. Neuroimage 36, 736-745.

Stephan, K.E., Penny, W.D., Moran, R.J., den Ouden, H.E., Daunizeau, J., Friston, K.J., 2010. Ten simple rules for dynamic causal modeling. Neuroimage 49, 3099-3109.

Urry, H.L., van Reekum, C.M., Johnstone, T., Kalin, N.H., Thurow, M.E., Schaefer, H.S., Jackson, C.A., Frye, C.J., Greischar, L.L., Alexander, A.L., Davidson, R.J., 2006. Amygdala and ventromedial prefrontal cortex are inversely coupled during regulation of negative affect and predict the diurnal pattern of cortisol secretion among older adults. J Neurosci 26, 4415-4425.

Vai, B., Bollettini, I., Benedetti, F., 2014. Corticolimbic connectivity as a possible biomarker for bipolar disorder. Expert Rev. Neurother. 14, 631-650.

Vai, B., Poletti, S., Radaelli, D., Dallaspezia, S., Bulgarelli, C., Locatelli, C., Bollettini, I., Falini, A., Colombo, C., Smeraldi, E., Benedetti, F., 2015a. Successful antidepressant chronotherapeutics enhance fronto-limbic neural responses and connectivity in bipolar depression. Psychiatry Res.

Vai, B., Sferrazza Papa, G., Poletti, S., Radaelli, D., Donnici, E., Bollettini, I., Falini, A., Cavallaro, R., Smeraldi, E., Benedetti, F., 2015b. Abnormal cortico-limbic connectivity during emotional processing correlates with symptom severity in schizophrenia. Eur Psychiatry.

Victor, T.A., Furey, M.L., Fromm, S.J., Bellgowan, P.S., Ohman, A., Drevets, W.C., 2012. The extended functional neuroanatomy of emotional processing biases for masked faces in major depressive disorder. PLoS One 7, e46439.

Victor, T.A., Furey, M.L., Fromm, S.J., Ohman, A., Drevets, W.C., 2010. Relationship between amygdala responses to masked faces and mood state and treatment in major depressive disorder. Arch Gen Psychiatry 67, 1128-1138.

Victor, T.A., Furey, M.L., Fromm, S.J., Ohman, A., Drevets, W.C., 2013. Changes in the neural correlates of implicit emotional face processing during antidepressant treatment in major depressive disorder.

International Journal of Neuropsychopharmacology 16, 2195-2208.

Volman, I., Verhagen, L., den Ouden, H.E., Fernandez, G., Rijpkema, M., Franke, B., Toni, I., Roelofs, K., 2013. Reduced serotonin transporter availability decreases prefrontal control of the amygdala. J Neurosci 33, 8974-8979.

Windischberger, C., Lanzenberger, R., Holik, A., Spindelegger, C., Stein, P., Moser, U., Gerstl, F., Fink, M., Moser, E., Kasper, S., 2010. Area-specific modulation of neural activation comparing escitalopram and citalopram revealed by pharmaco-fMRI: a randomized cross-over study. Neuroimage 49, 1161-1170. Wu, J., Buchsbaum, M.S., Gillin, J.C., Tang, C., Cadwell, S., Wiegand, M., Najafi, A., Klein, E., Hazen, K., Bunney, W.E., Jr., Fallon, J.H., Keator, D., 1999. Prediction of antidepressant effects of sleep deprivation by metabolic rates in the ventral anterior cingulate and medial prefrontal cortex. Am J Psychiatry 156, 11491158.

Wu, J.C., Buchsbaum, M., Bunney, W.E., Jr., 2001. Clinical neurochemical implications of sleep deprivation's effects on the anterior cingulate of depressed responders. Neuropsychopharmacology 25, S74-78. 
Yoo, S.Y., Jang, J.H., Shin, Y.W., Kim, D.J., Park, H.J., Moon, W.J., Chung, E.C., Lee, J.M., Kim, I.Y., Kim, S.I., Kwon, J.S., 2007. White matter abnormalities in drug-naive patients with obsessive-compulsive disorder: a diffusion tensor study before and after citalopram treatment. Acta Psychiatr. Scand. 116, 211-219. 


\section{Figure Legends}

Figure 1. On the top: schematic graphic representation of model space for DCM analyses, composed by 16 competitive models for DCM. Yellow arrows represent driving inputs: fearful faces enter the network from FG and Amy. Fixed intrinsic connections (continues arrows) have been modeled in all DCMs: from FG to Amy, and bidirectionally between Amy, ACC and VLPFC. The 16 DCM models varied for the presence or absence of modulatory effects on the connections between PFC (specifically ACC and VLPFC) and Amy. The dashed arrows represent the parameters that varied across the models. On the bottom: brain regions significantly activated by the task (one sample t-test). Data are shown for the main effects surviving a threshold of $\mathrm{P}<0.01$ in the whole brain.

Figure 2. Graphic representation of the significant interaction between response to treatment and time: nRMDD achieved a significant improvement after 5/6 weeks (Post hoc test: final scores vs pre-treatment in nRMDD, $\left.{ }^{*} p<0.05\right)$, even not reaching clinical remission, while responders showed a progressive amelioration during the treatment (intermediate scores vs pre-treatment ${ }^{*} p<0.05$; final scores $* * p<0.0001$ both vs pre-treatment and vs non responders).

Figure 3. Graphic representation of DCMs results. Dashed arrows represent the DCM parameters that were different at the pre-treatment or changed after the treatment in patients. Yellow arrows symbolize the driving inputs. nRMDD showed a significant reduced endogenous connections from Amy to ACC and from Amy to VLPFC compared to HC. These connections are inhibitory in nRMDD, and excitatory in other groups. nRMDD had an increased positive connectivity (modulation plus endogenous connectivity) and excitatory modulatory effect from ACC to Amy compared to $\mathrm{HC}$, who instead presented an inhibitory top-down control from ACC to Amy. Whiskers indicate $95 \%$ Confidence Intervals; $* p<0.05$ 